\title{
Is Uniqueness Lost for Under-Sampled Continuous-Time Auto-Regressive Processes?
}

\author{
John Paul Ward, Hagai Kirshner, and Michael Unser, Fellow, IEEE
}

\begin{abstract}
We consider the problem of sampling continuous-time auto-regressive processes on a uniform grid. We investigate whether a given sampled process originates from a single continuous-time model, and address this uniqueness problem by introducing an alternative description of poles in the complex plane. We then utilize Kronecker's approximation theorem and prove that the set of non-unique continuous-time AR(2) models has Lebesgue measure zero in this plane. This is a key aspect in current estimation algorithms that use sampled data, as it allows one to remove the sampling rate constraint that is imposed currently.
\end{abstract}

Index Terms-Approximation theory, sampling theory, stochastic processes.

\section{INTRODUCTION}

C ONTINUOUS-TIME ARMA (Auto Regressive Moving Average) processes are widely used in control theory and in signal processing and analysis. Typical examples of applications are system identification and adaptive filtering [1], [2]. as well as speech analysis and synthesis [3]. In practice, the available data is discrete and one is required to estimate the underlying continuous-domain parameters from sampled data.

Focusing on direct estimation approaches, they often associate the discretization process with loss of information. This means that two continuous-time processes can result in two equivalent discrete-time processes that share the same autocorrelation sequence. In order to avoid such ambiguity, some of them assume a relatively high sampling rate [4]-[14]. This non-uniqueness property originates from the fact that the power spectrum function of a continuous-time ARMA model is not band-limited, and from the fact that the discrete-time pole is invariant to $2 \pi j k$ shifts of the continuous-time pole in the complex plane [10].

Nevertheless, it seems that the special structure of the continuous-time ARMA power spectrum function has been overlooked within this context. The continuous-time power spectrum is a rational function of two symmetric polynomials in the Laplace domain, and it becomes a rational function of yet another two symmetric polynomials in the $z$-domain upon sampling. This mapping could potentially be invertible. Indeed, the

Manuscript received November 01, 2011; revised January 10, 2012; accepted January 18, 2012. Date of publication January 23, 2012; date of current version February 09, 2012. This work was supported in part by the Swiss National Science Foundation under Grant 200020-121763 and by the ERC Grant ERC-2010-AdG 267439-FUN-SP. The associate editor coordinating the review of this manuscript and approving it for publication was Prof. Saeid Sanei.

The authors are with EPFL, STI, Lausanne CH-1015, Switzerland.

Color versions of one or more of the figures in this paper are available online at http://ieeexplore.ieee.org.

Digital Object Identifier 10.1109/LSP.2012.2185695 discrete-time poles are invariant to certain shifts in the complex plane, but the discrete-time zeros are not.

Using the structure of the continuous-time power spectrum to analyze uniqueness of continuous-time AR (Auto Regressive) models is important because it could remove the sampling rate constraint used in current estimation algorithms. This could be instrumental to direct estimation methods that restrict the sampling interval value from being too large, as in the preceding references. Continuous-time AR(1) and AR(2) models are of central importance in describing a general continuous-time AR model of simple poles. We focus on the latter and consider the problem of sampling a continuous-time AR(2) process on a unit-interval grid. The autocorrelation sequence of the sampled process is then given by the values of the original autocorrelation function on the very same grid. The question we are raising here is whether or not the sampling process is a one-to-one mapping of the continuous-time model to its discrete-time counterpart, and we aim at identifying the set of parameters for which the sampling operator is invertible. We address this problem by introducing an alternative description of the continuous-time AR model in the complex plane and by utilizing Kronecker's approximation theorem for describing uniform sampling of periodic functions.

\section{Alternative Description of Poles}

The bilateral Laplace transform of a scalar function $\varphi(t)$ is

$$
\Phi(s)=\int_{-\infty}^{\infty} \varphi(t) e^{-s t} d t,
$$

where $s$ takes complex values that satisfy $\int_{-\infty}^{\infty}\left|\varphi(t) e^{-s t}\right| d t<$ $\infty$. The Fourier transform of this function is $\hat{\varphi}(\omega)=\Phi(j \omega)$. The complex conjugate of $c$ is $c^{*}$. In this work, $\varphi(t)$ is an autocorrelation function and $\hat{\varphi}(\omega)$ is the corresponding power spectrum. A continuous-time AR(1) model is described by two parameters: the single real pole and the intensity of innovation process. The pole should be strictly negative. A continuous-time AR(2) model can be described by three parameters: the intensity of the innovation process and two possibly complex poles. If one of the poles is real then so is the other. If one of the poles is complex then the other is its complex conjugate. In any case, both poles must have strictly negative real values. The coupling between the two poles prevents them from being described by two arbitrary points in the complex plane. We therefore suggest an alternative description that requires only a single point (Fig. 1). Let $c=c_{1}+j c_{2}$ be a complex number. Then

$$
\begin{aligned}
& D_{1}:=\left\{c \in \mathbb{C}: c_{1}=c_{2}<0\right\} \\
& D_{2}:=\left\{c \in \mathbb{C}: c_{1}<c_{2}<0\right\} \\
& D_{3}:=\left\{c \in \mathbb{C}: c_{1}<0, c_{2}>0\right\}
\end{aligned}
$$


TABLE I

An Alternative Description of Poles in the Complex Plane for a Continuous-Time AR(2) Model

\begin{tabular}{|c|c|c|c|}
\hline Region & Poles & $\Phi(\mathbf{s} ; \boldsymbol{\theta})$ & $\varphi(\mathbf{t} ; \theta), \mathbf{t} \geq 0$ \\
\hline$D_{1}$ & single multiple real pole & $\frac{\sigma^{2}}{\left(s-c_{1}\right)^{2}\left(-s-c_{1}\right)^{2}}$ & $\frac{\sigma^{2}}{4 c_{1}^{3}}\left(c_{1} t-1\right) e^{c_{1} t}$ \\
\hline$D_{2}$ & two distinct real poles & $\frac{\sigma^{2}}{\left(s-c_{1}\right)\left(-s-c_{1}\right)\left(s-c_{2}\right)\left(-s-c_{2}\right)}$ & $\frac{\sigma^{2}}{2\left(c_{1}^{2}-c_{2}^{2}\right)}\left(\frac{1}{c_{1}} e^{c_{1} t}-\frac{1}{c_{2}} e^{c_{2} t}\right)$ \\
\hline$D_{3}$ & two complex conjugate poles & $\frac{\sigma^{2}}{(s-c)(-s-c)\left(s-c^{*}\right)\left(-s-c^{*}\right)}$ & $\frac{\sigma^{2}}{4 c_{1}\left(c_{1}^{2}+c_{2}^{2}\right)}\left(-\cos \left(c_{2} t\right)+\frac{c_{1}}{c_{2}} \sin \left(c_{2} t\right)\right) e^{c_{1} t}$ \\
\hline
\end{tabular}

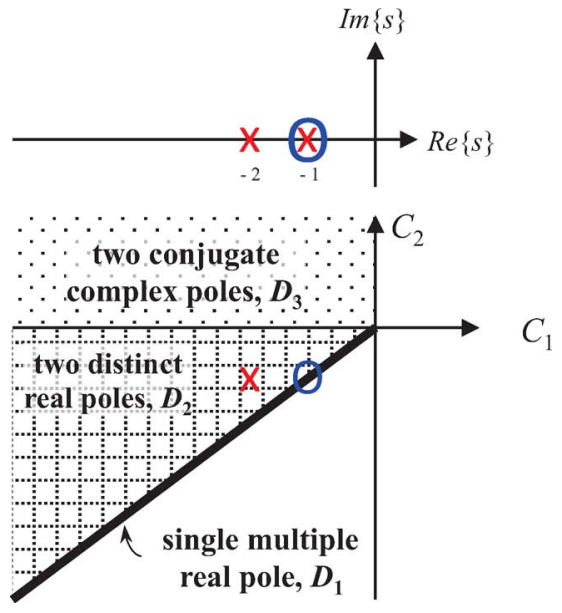

Fig. 1. An alternative description of poles for a continuous-time AR(2) process in the complex plane. The standard description is shown at the top. The red ' $x$ ' marks represent a process having two real values $s_{1}=-1, s_{2}=-2$. The blue circle represents a process having two multiple poles at $s_{1,2}=-1$. The two processes share a single pole at $s=-1$ and the corresponding location is re-used in the standard description. Such a re-use does not occur in the proposed alternative description, as depicted at the bottom.

We assign a point in $D_{1}$ to represent a continuous-time process with a single multiple real pole, $D_{2}$ to represent two distinct real poles, and $D_{3}$ to represent two complex conjugate poles. Any point in $D=D_{1} \cup D_{2} \cup D_{3}$ defines a valid continuous-time $\mathrm{AR}(2)$ process as shown in Table I, and all possible continuous-time $\mathrm{AR}(2)$ processes are represented by the points in $D$. Such a description allows us to associate a single point in the complex plane with a single continuous-time AR(2) process, and this property is useful for assigning a measure to the collection of non-unique processes. In the standard representation, such an analysis is impeded by the fact that any given point on the real axis is used in the description of multiple processes (Fig. 1).

\section{The ProBlem}

Definition 1: Let $c<0$ and $\sigma_{c}^{2}>0$. Then, $\theta=\left(\sigma_{c}^{2}, c\right)$ is a vector of parameters for a continuous-time $\mathrm{AR}(1)$ model.

Definition 2: Let $c \in D$ and $\sigma_{c}^{2}>0$. Then, $\theta=\left(\sigma_{c}^{2}, c\right)$ is a vector of parameters for a continuous-time $\operatorname{AR}(2)$ model.

Definition 3: $\theta_{1}$ and $\theta_{2}$ are equivalent if $\varphi\left(n ; \theta_{1}\right)=\alpha$. $\varphi\left(n ; \theta_{2}\right), n \in \mathbb{Z}^{+} \cup\{0\}, \alpha \in \mathbb{R}$ according to Table I. If $\theta_{1}$ has no equivalent $\theta_{2}$, then it is unique.

We are concerned with identifying the set of unique continuous-time AR models and with the construction of equivalent vectors of parameters. For example, the continuous-time AR(2)
TABLE II

Possible Cases of Equivalent Pairs in $D$

\begin{tabular}{c|ccc}
\hline \hline & $D_{1}$ & $D_{2}$ & $D_{3}$ \\
\hline$D_{1}$ & A & B & C \\
$D_{2}$ & & D & E \\
$D_{3}$ & & & F \\
\hline \hline
\end{tabular}

processes $\theta_{k}=(1,-1+2 \pi k j)$ are equivalent for $k \in \mathbb{Z}^{+}$. On the other hand, every continuous-time $\operatorname{AR}(2)$ processes $\theta_{k}=$ $(1,-1+(2+2 \pi k) j)$ is unique.

\section{Uniqueness of SAmpled AR(1) And AR(2) Processes}

Theorem 1: Every continuous-time $\mathrm{AR}(1)$ process is unique.

Proof: The autocorrelation function of a continuous-time $\operatorname{AR}(1)$ model is $\varphi(t ; \theta)=\sigma_{c}^{2} \cdot e^{c|t|}$. The equivalence property translates in this case to $\sigma_{c 1}^{2} \cdot e^{c 1|t|}-\sigma_{c 2}^{2} \cdot e^{c 2|t|}$. This is impossible if the two functions decay at different rates.

Our approach to the AR(2) problem relies on the different forms of the autocorrelation function of Table I. There are six pairs that should be considered when determining uniqueness (Table II), and we examine each one of them separately.

Case A: Let $c, d \in D_{1}$. The equivalence property translates in this case to the following equation

$$
\frac{\sigma_{c}^{2}}{4 c_{1}^{3}}\left(c_{1} n-1\right) e^{c_{1} n}-\frac{\sigma_{d}^{2}}{4 d_{1}^{3}}\left(d_{1} n-1\right) e^{d_{1} n}=0 .
$$

More generally

$$
\alpha\left(1-c_{1} n\right) e^{c_{1} n}+\beta\left(1-d_{1} n\right) e^{d_{1} n}=0 .
$$

As $c_{1} \neq d_{1}$, the two exponential terms have different rates of decay. Therefore, the sampled versions of such functions cannot be made equal by adjusting the scalars $\alpha, \beta$.

Case B: Let $c \in D_{1}$ and $d \in D_{2}$. The equivalence property translates in this case to the following linear combination

$$
\alpha\left(1-c_{1} n\right) e^{c_{1} n}+\beta e^{d_{1} n}+\gamma e^{d_{2} n}=0 .
$$

Alternatively

$$
\alpha\left(1-c_{1} n\right)+\beta e^{\left(d_{1}-c_{1}\right) n}+\gamma e^{\left(d_{2}-c_{1}\right) n}=0 .
$$

If $d_{1} \neq d_{2} \neq c_{1}$, then the two exponential functions need to cancel a monotonic linear function. Each of them is either decaying or growing and their sum cannot cancel the linear term. A similar argument applies to the case where one of the exponentials becomes a constant, e.g. $d_{1}-c_{1}=0$. 


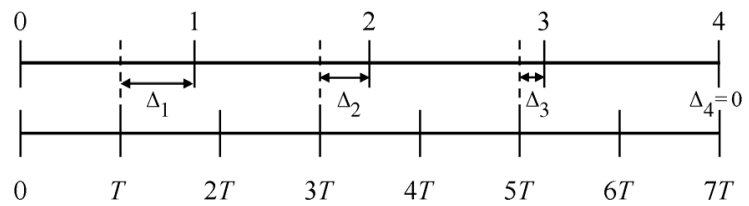

Fig. 2. Kronecker's approximation theorem applied to a periodic function. The period of the function is $T$ and we sample it on a unit-interval grid. The values at the sampling points are given by the values of the function at locations $\Delta_{n}$ where $n=0,1,2, \ldots$ According to the theorem, if $T$ is irrational, then the sequence $\left\{\Delta_{n}\right\}$ is dense in $[0, T]$.

In the following, $\|x\|$ denotes the distance to the greatest integer smaller than or equal to $x$.

Theorem (Kronecker's Approximation Theorem [15]):

1) If the real numbers $\left(x_{1}, \ldots, x_{n}\right)$ are linearly independent over $\mathbb{Q}$, then for real $\alpha_{k}$ and $\epsilon>0$ there are arbitrarily large real $t$ for which

$$
\left\|t x_{k}-\alpha_{k}\right\|<\epsilon \quad k=1, \ldots, n .
$$

2) If the numbers $\left(1, x_{1}, \ldots, x_{n}\right)$ are linearly independent over $\mathbb{Q}$, then in part $1, t$ can be taken to be an arbitrarily large integer.

Case $C$ : Let $c \in D_{1}$ and $d \in D_{3}$. Similar to the previous case, we have

$$
\alpha\left(1-c_{1} n\right)+\beta\left(\cos \left(d_{2} n\right)-\frac{d_{1}}{d_{2}} \sin \left(d_{2} n\right)\right) e^{\left(d_{1}-c_{1}\right) n}=0 .
$$

It might happen that the sampled version of the periodic term will converge to zero at a rate that would compensate for the exponential $e^{\left(d_{1}-c_{1}\right) n}$. If the compensation matches the linear term $1-c_{1} n$, then $c$ and $d$ would be an equivalent pair. This, however, cannot happen. If $d_{1}-c_{1}<0$, then the exponential term is decaying, hence it cannot be canceled by the linear term. If $d_{1}-c_{1}>0$ then we examine the period $T=2 \pi / d_{2}$. If $T$ is irrational, then by Kronecker's approximation theorem the unit-interval sampling grid is dense in the $T$ grid (Fig. 2), and there is a sequence $n_{k}$ such that $\cos \left(d_{2} n_{k}\right)-d_{1} / d_{2} \sin \left(d_{2} n_{k}\right)$ converges to a non-zero value. This means that for those sampling points, the linear term cannot cancel the exponential growth. If $T$ is rational, it means that the periodic term takes finitely many values. If one of these values is non-zero, then we have a subsequence for which the periodic term is constant; the linear term cannot cancel the exponential growth for this subsequence either. If all the sample values are zero, then the linear term should be zero on the sampling grid, too. This is a contradiction, as $1-c_{1} n$ is zero for at most a single value of $n$.

Case D: Let $c, d \in D_{2}$. The equivalence property translates in this case to the following linear combination

$$
\alpha e^{c_{1} n}+\beta e^{c_{2} n}+\gamma e^{d_{1} n}+\delta e^{d_{2} n}
$$

Since the pairs $c, d$ are distinct, there must be one function in the the sum with a non-comparable rate of decay. Also, a finite linear combination of exponentials is a Haar space on $\mathbb{R}$, and any such linear combination can have at most a finite number of zeros.
Case E: Let $c \in D_{2}$ and $d \in D_{3}$. The equivalence property translates in this case to the following linear combination

$$
\alpha e^{c_{1} n}+\beta e^{c_{2} n}+\gamma\left(\cos \left(d_{2} n\right)-\frac{d_{1}}{d_{2}} \sin \left(d_{2} n\right)\right) e^{d_{1} n}=0 .
$$

This can be written as

$\alpha e^{\left(c_{1}-d_{1}\right) n}+\beta e^{\left(c_{2}-d_{1}\right) n}+\gamma\left(\cos \left(d_{2} n\right)-\frac{d_{1}}{d_{2}} \sin \left(d_{2} n\right)\right)=0$.

If one of the exponentials is growing, then the linear combination cannot be made zero. If both exponentials are decaying, then it might happen that the periodic term will cancel the term $\alpha e^{\left(c_{1}-d_{1}\right) n}+\beta e^{\left(c_{2}-d_{1}\right) n}$ after all. This, however, cannot happen and we apply the same reasoning as in Case $\mathrm{C}$.

Case $F$ : Let $c, d \in D_{3}$. The equivalence property translates in this case to the following linear combination

$$
\begin{aligned}
& \alpha\left(\cos \left(c_{2} n\right)-\frac{c_{1}}{c_{2}} \sin \left(c_{2} n\right)\right) e^{c_{1} n} \\
& \quad+\beta\left(\cos \left(d_{2} n\right)-\frac{d_{1}}{d_{2}} \sin \left(d_{2} n\right)\right) e^{d_{1} n}=0 .
\end{aligned}
$$

We need additional assumptions on $c_{2}$ to prove uniqueness. Consider for example $c_{1}=d_{1}=-1, c_{2}=2 \pi$, and $d_{2}=4 \pi$. The sampled version of the two autocorrelation functions are the same in this case although $c \neq d$. We will therefore focus on cases where $c_{2}$ is an irrational multiple of $\pi$, making the period of the trigonometric term irrational. If $c_{1} \neq d_{1}$ then we can apply the same reasoning as in Cases $\mathrm{C}, \mathrm{E}$ and show that it is not possible to have two equivalent process. If $c_{1}=d_{1}$, then the two exponentials have the same rate of decay. The question, then, is whether the sampled versions of the two periodic terms can be made equal when $c_{2} \neq d_{2}$. We address this matter by utilizing Kronecker's approximation theorem, as shown next.

\section{A. Periodic Functions on a Grid}

Definition 4: $T(c)=2 \pi / c_{2}$ is the period of

$$
\phi(t ; c)=\cos \left(c_{2} t\right)-\frac{c_{1}}{c_{2}} \sin \left(c_{2} t\right)
$$

where $c=c_{1}+j c_{2}$ is in $D_{3}$ and $t \geq 0$.

Definition 5: Let $\{k T(c)\}_{k \in \mathbb{Z}^{+} \cup\{0\}}$ be a grid. The distance of $n \in \mathbb{Z}^{+}$from this grid is defined by (Fig. 2)

$$
\Delta_{n}(c)=\min _{k \in \mathbb{Z}^{+} \cup\{0\}}\{n-k T(c)\} \text { s.t. } n \geq k T(c) .
$$

If $T(c)$ is rational, then the sequence $\left\{\Delta_{n}(c)\right\}_{n}$ takes finitely many different values. If $T(c)$, however, is irrational, then according to Kronecker's approximation theorem the sequence is dense in $[0, T(c)]$. We consider next the case of two sampled periodic functions.

Proposition 1: Let $T(c)$ and $T(d)$ be irrational such that $1,1 / T(c)$ and $1 / T(d)$ are independent over $\mathbb{Q}$. Then, the set $\left\{\left(\Delta_{n}(c), \Delta_{n}(d)\right)\right\}_{n \in \mathbb{Z}^{+}}$is dense in $[0, T(c)] \times[0, T(d)]$. 
As an example, if $((1 / T(c)),(1 / T(d)), 1)=(\sqrt{3}, \sqrt{2}, 1)$, then the equivalence property translates here into the requirement that $\phi\left(t_{1}, c\right)=\phi\left(t_{2}, d\right)$ for any pair $\left(t_{1}, t_{2}\right)$ in $[0, \sqrt{3}] \times$ $[0, \sqrt{2}]$. Since $\phi(t ; \cdot)$ is not a constant function, there is no way of meeting this requirement. Other examples are $\left(\pi, \pi^{2}, 1\right)$ and $(\pi, e, 1)$. We note that $(\pi,(\pi+1 / 3), 1)$ are linearly dependent over the rationals and for such cases we introduce the next two propositions.

Proposition 2: Let $T(c)$ and $T(d)$ be irrational such that $\left(n_{2} / T(d)\right)=\left(n_{1} / T(c)\right)+n_{3}$ for some $n_{1}, n_{2}, n_{3} \in \mathbb{N}$. Then, the closure of the set $\left\{\left(\Delta_{n}(c), \Delta_{n}(d)\right)\right\}_{n \in \mathbb{Z}^{+}}$contains one of the following lines

$$
\begin{cases}y=\frac{n_{1}}{n_{2}} \frac{T(d)}{T(c)} \cdot x & \frac{n_{1}}{n_{2}}>0 \\ y=T(d)+\frac{n_{1}}{n_{2}} \frac{T(d)}{T(c)} \cdot x & \frac{n_{1}}{n_{2}}<0\end{cases}
$$

under the quotient map

$$
\left\{\begin{array}{l}
\Delta(c)=x-T(c)\left\lfloor\frac{x}{T(c)}\right\rfloor \\
\Delta(d)=y-T(d)\left\lfloor\frac{y}{T(d)}\right\rfloor
\end{array} .\right.
$$

Proposition 3: Let $T(c)$ be irrational and let $T(d)$ be rational. Then, the closure of the set $\left\{\left(\Delta_{n}(c), \Delta_{n}(d)\right)\right\}_{n \in \mathbb{Z}^{+}}$contains a line that passes through $(0,0)$ with slope zero.

Under the conditions of Proposition 3, the equivalence property translates into the requirement that $\phi(t ; c)=\phi(0 ; d)$. This cannot happen since $\phi(t ; \cdot)$ is not a constant function. As for Proposition 2 , it still requires $\phi(\cdot ; c)$ to be equal to $\phi(\cdot ; d)$ on a line. This line, however, has a more involved structure, and this requirement implies $c=d$.

Case F (Contd.): Propositions 1-3 mean that if $T(c)$ is irrational, then there are no equivalent points in $D_{3}$. As $T(c)=$ $2 \pi / c_{1}$, it follows that if $c$ is not a rational multiple of $\pi$, then it is unique in $D_{3}$.

Our analysis gives rise to the following theorem which quantifies the prevalence of continuous-time $\mathrm{AR}(2)$ processes for which there exists a unique set of parameters that comply with the sampled version of the autocorrelation function.

Theorem 2: Almost every continuous-time AR(2) process is unique. Specifically, the set of non-unique continuous-time $\mathrm{AR}(2)$ processes has Lebesgue measure zero in the complex plane.

All continuous-time $\mathrm{AR}(2)$ processes that are defined by Cases A, B, C, D, and E are unique. Ambiguity may arise only in Case $\mathrm{F}$ which compares two processes that have a set of two conjugate complex poles. The autocorrelation function in this case includes a periodic term that is sampled on the unit grid. Propositions 1-3 state that if the period is irrational, then uniqueness is guaranteed. If it is rational, then there might be cases in which the sampled versions of the periodic terms will differ by a constant multiplicative value that can be associated with the innovation intensity $\sigma^{2}$. This is in agreement with previous known results that avoid any possible ambiguity by restricting the imaginary part of the continuous-time poles. Theorem 2 shows, however, that the Lebesgue measure of such ambiguous continuous-time processes is zero in the complex plane, and that there are more continuous-time $\mathrm{AR}(2)$ processes which are uniquely defined by their sampled version. We note that additional knowledge on $\sigma^{2}$ may solve the ambiguity completely. Another possible way is to consider more than one sampling grid.

Theorem 2 implies that sampling rate values do not impose limitations on parameters estimation from sampled data. Currently available methods assume sampling rate values that are relatively high compared to the time constant of the model, and such an assumption is no longer required. This, in turn, opens up new opportunities for estimation algorithms that take the sample values of the autocorrelation function into account as was recently done in [16].

\section{CONCLUSIONS}

In this work, we considered sampling of continuous-time AR models on a uniform grid. We investigated whether the discrete-domain model is unique in the sense that it originates from a single continuous-time model. We focused on the continuoustime $\mathrm{AR}(2)$ process and introduced an alternative description of poles in the complex plane. Such a description avoids the coupling between real and complex poles. We then utilized Kronecker's approximation theorem for proving that the set of nonunique continuous-time $\mathrm{AR}(2)$ models has Lebesgue measure zero. This result allows one to remove the sampling rate constraint that is used in currently available estimation algorithms, and to derive improved algorithms that overcome the power spectrum aliasing.

\section{REFERENCES}

[1] L. Ljung, System Identification. Upper Saddle River, NJ: PrenticeHall, 1999.

[2] S. Haykin, Adaptive Filter Theory. Upper Saddle River, NJ: PrenticeHall, 2002.

[3] I.-T. Lim and B. G. Lee, "Lossless pole-zero modeling for speech signals," IEEE Trans. Speech Audio Process., vol. 1, no. 3, pp. 269-276, Jul. 1993.

[4] A. Feuer and G. Goodwin, Sampling in Digital Signal Processing and Control. Boston, MA: Birkhäuser, 1996.

[5] H. Fan, T. Söderström, M. Mossberg, B. Carlsson, and Y. Zou, "Estimation of continuous-time AR process parameters from discrete-time data," IEEE Trans. Signal Process., vol. 47, no. 5, pp. 1232-1244, May 1999.

[6] D.-T. Pham, "Estimation of continuous-time autoregressive model from finely sampled data," IEEE Trans. Signal Process., vol. 48, no. 9, pp. 2576-2584, Sep. 2000.

[7] H. Garnier, M. Mensler, and A. Richard, "Continuous-time model identification from sampled data: Implementation issues and performance evaluation," Int. J. Contr., vol. 76, no. 13, pp. 1337-1357, Sep. 2003.

[8] H. Garnier and P. Young, "Time-domain approaches to continuoustime model identification of dynamical systems from sampled data," in Proc. Amer. Control Conf., Boston, MA, June 2004.

[9] G. C. Goodwin, J. I. Yuz, and H. Garnier, "Robustness issues in continuous-time system identification from sampled data," in Proc. 16th IFAC World Congress, Prague, Czech Republic, Jul. 2005.

[10] E. K. Larsson, M. Mossberg, and T. Söderström, "An overview of important practical aspects of continuous-time ARMA system identification," Circuits Syst. Signal Process., vol. 25, no. 1, pp. 17-46, May 2006.

[11] H. Garnier and L. Wang, Eds., Identification of Continuous-Time Models from Sampled Data. London, U.K.: Springer-Verlag, 2008.

[12] G. C. Goodwin, J. C. Agäero, J. S. Welsh, J. I. Yuz, G. J. Adams, and C. R. Rojas, "Robust identification of process models from plant data," J. Process Contr., vol. 18, pp. 810-820, 2008.

[13] M. Mossberg, "Estimation of continuous-time stochastic signals from sample covariances," IEEE Trans. Signal Process., vol. 56, no. 2, pp. 821-825, Feb. 2008.

[14] J. Gillberg and L. Ljung, "Frequency-domain identification of continuous-time ARMA models from sampled data," Automatica, vol. 45, pp. 1371-1378, 2009

[15] H. Bohr and B. Jessen, "One more proof of Kronecker's theorem," $J$. London Math. Soc., vol. 7, no. 4, pp. 274-275, 1932.

[16] H. Kirshner, S. Maggio, and M. Unser, "A sampling theorey approach to continuous ARMA identification," IEEE Trans. Signal Process., vol. 59, no. 10, pp. 4620-4634, Oct. 2011. 